\title{
Relação entre 0 grau de fragilidade de idosas e a realização de exames preventivos
}

\author{
Thais Debona Crespi", Dinara Hansen", Solange Beatriz Billig Garces "**, Carolina Böettge Rosa ****,

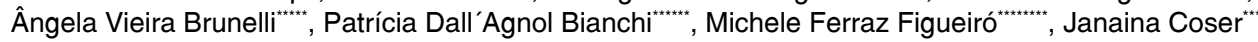

\section{Resumo}

A população idosa é a parcela que mais cresce no Brasil e no mundo, e a prevenção contra os cânceres de mama e de colo do útero em idosas não pode ser desprezada, pois essa é a parcela da população mais vulnerável. Assim, é importante verificar se existe associação entre o grau de fragilidade e a realização de exames preventivos do câncer de colo do útero e da mama em idosas. Os dados foram coletados por meio de entrevista estruturada, com idosas de 60 anos ou mais, residentes no município de Cruz Alta. O questionário utilizado continha dados sociodemográficos, detecção precoce e previsibilidade de agravos e a realização de exames preventivos. A análise estatística dos dados foi feita com o auxílio do software IBM SPSS 20. A amostra do presente estudo foi constituída por 343 mulheres, das quais $62,1 \%$ apresentaram baixo risco de adoecer, $8,16 \%$, risco mé- dio, $15,74 \%$, risco médio-alto, e $14 \%$, risco alto. Quanto às recomendações, 79,59\% receberam orientação sobre a importância da realização dos exames preventivos, embora $28,57 \%$ nunca tenham feito o Papanicolau, $37,90 \%$ nunca fizeram o autoexame de mamas, 38,19\% nunca fizeram o exame clínico das mamas, e 39,07\% nunca fizeram mamografia. Não foi encontrada associação significativa entre as variáveis grau de fragilidade e exames preventivos realizados pelas idosas. Todavia, é importante a continuidade e a ampliação das estratégias de educação em saúde sobre a detecção precoce dessas doenças, sobretudo na população idosa, de forma a garantir a adesão dessas mulheres a esse cuidado preventivo.

Palavras-chave: Idoso. Fragilidade. Prevenção. Mamografia. Papanicolau.

* Acadêmica do curso de Biomedicina. Bolsista do Grupo Interdisciplinar de Estudos em Envelhecimento Humano (Unicruz). E-mail: thaiscrespi@hotmail.com

** Fisioterapeuta. Mestre em Gerontologia Biomédica - PUCRS. Docente e pesquisadora do Grupo Interdisciplinar de Estudos em Envelhecimento Humano (Unicruz). E-mail: dinarahansen@hotmail.com

**** $\quad$ Educadora física. Doutora em Ciências Sociais (Unisinos). Docente e pesquisadora do Grupo Interdisciplinar de Estudos em Envelhecimento Humano (Unicruz). E-mail: sgarces@unicruz.edu.br Nutricionista. Mestre em Patologia (UFCSPA). Docente e pesquisadora do Grupo Interdisciplinar de Estudos em Envelhecimento Humano (Unicruz). E-mail: carolboettge@gmail.com

Nutricionista. Mestre em Extensão Rural (UFSM). Docente e pesquisadora do Grupo Interdisciplinar de Estudos em Envelhecimento Humano (Unicruz). E-mail: angelavbrunelli@gmail.com

Fisioterapeuta. Doutora em Fisiologia (Ufrgs). Docente e pesquisadora do Grupo Interdisciplinar de Estudos em Envelhecimento Humano (Unicruz). E-mail: patibianchi@yahoo.com.br

Bacharel em Física. Pós-Doutorado em Estatística Aplicada (USP). Docente e pesquisadora do Grupo Interdisciplinar de Estudos em Envelhecimento Humano (Unicruz). E-mail: mferrazfigueiro@gmail.com Biomédica. Doutora em Biologia Celular e Molecular Aplicada à Saúde (Ulbra). Docente e pesquisadora do Grupo Interdisciplinar de Estudos em Envelhecimento Humano (Unicruz). Autora correspondente. E-mail: coser@unicruz.edu.br

$\rightarrow$ http://dx.doi.org/10.5335/rbceh.2014.4223

Recebido em: 05/09/2014. Aceito em: 27/04/2015 


\section{Introdução}

A população idosa é a parcela que mais cresce no Brasil, assim como no mundo todo (VERAS, 2012). Comparando-se dados de 2010 e de 2013, percebe-se que esse aumento ocorre de forma cada vez mais acelerada. O censo de 2010 apontou que mais de 18 milhões da população brasileira eram de idosos, e conforme dados da Pesquisa Nacional por Amostra de Domicílios (PNAD), em 2012 já eram aproximadamente 24 milhões (IBGE, 2010, 2012).

Esse crescimento da população idosa altera não só a pirâmide etária como também o contexto e a organização do serviço de saúde nacional. Observa-se um crescimento na ocorrência e no prolongamento de diversas patologias, o que acarreta o aumento do cuidado com o idoso, de tratamentos médicos e hospitalares, de fornecimento de medicamentos, consequentemente, das despesas públicas (VERAS, 2003).

Mesmo diante da senilidade, a prevenção não deve ser descartada. Idosos fragilizados, com problemas crônicos de saúde e incapacidades, quando identificados tardiamente, dificultam a aplicação de intervenções que possam subverter ou minimizar os problemas, o que mostra a importância da prevenção (OLIVEIRA; MENEZES, 2011).

Segundo Oliveira e Menezes (2011), de um modo geral, o avanço da idade está associado ao maior risco de ocorrência da síndrome da fragilidade. $\mathrm{E}$, apesar de não haver, atualmente, uma concordância a respeito do que é a fragilidade e de quem é o idoso frágil, é consensual que o idoso fragilizado apresenta alterações biológicas, psicológicas e sociais correlacionadas umas com as outras de maneira complexa (TRIBESS; OLIVEIRA, 2011).
Estudos realizados no país demostram que os idosos apresentam, na maioria dos casos, mais de uma doença crônica. Devido, em parte, pelo envelhecimento trazer mudanças nas células que aumentam sua suscetibilidade à transformação maligna. Além disso, as células das pessoas idosas foram expostas por mais tempo a possíveis fatores de risco cancerígenos ou carcinógenos, como tabagismo, alcoolismo, hábitos alimentares e radiação solar (DA SILVA, M. M.; DA SILVA, V. H., 2005).

No ano de 2007, as principais causas de mortalidade de idosos no Brasil foram doenças do aparelho circulatório, seguido das neoplasias, as doenças infecciosas e parasitárias ocuparam a oitava posição (BRASIL, 2010).

Dentre as neoplasias mais incidentes nas mulheres brasileiras, destacam-se 0 câncer de mama e o câncer de colo do útero, para os quais estimam-se 57 mil e 15 mil novos casos, respectivamente, para o ano de 2014 (INCA, 2013). Ressalta-se, no entanto, que esses cânceres podem ser diagnosticados ainda no seu início, pelos exames conhecidos por preventivos, entre os quais se destacam o exame clínico das mamas, a mamografia e o citopatológico do colo do útero, mais conhecido como Papanicolau ou preventivo (INCA, 2011). Esses procedimentos estão disponíveis na rede do Sistema Único de Saúde (SUS), e podem diagnosticar esses agravos nas mulheres, permitindo uma intervenção rápida e adequada, tornando-as menos vulneráveis.

Sendo assim, verificar se existe dependência entre as variáveis grau de fragilidade e exames preventivos de câncer de colo do útero e de mama, no âmbito de informação e realização, é o objetivo deste trabalho. 


\section{Método}

O presente estudo integra o projeto intitulado Identificação precoce e previsibilidade de agravos em população idosa atendida pelas Estratégias de Saúde da Família nos municípios integrantes do Corede Alto Jacuí, com financiamento do edital PPSUS/Fapergs. Caracteriza-se como uma investigação observacional, descritiva, transversal, com uma abordagem quantitativa, desenvolvida pelo Grupo Interdisciplinar de Estudos em Envelhecimento Humano (GIEEH) da Unicruz, em idosas de 60 anos ou mais, residente no município de Cruz Alta. Segundo dados do IBGE (2010), esse município tem de 9.001 idosos, dos quais, 5.312 são mulheres. A amostra desta pesquisa foi obtida do banco de dados do citado projeto e é constituída por 343 mulheres acima de 60 anos e residentes no município de Cruz Alta. A pesquisa passou pela apreciação do Comitê de Ética em Pesquisa (CEP) Unicruz e obteve aprovação sob Certificado de Apresentação para Apreciação Ética (CAAE) no 0060.0.0.417.000-09.

Foram realizadas visitas domiciliares no ano de 2012, e as idosas responderam a um instrumento que continha dados sociodemográficos. Para avaliar o grau de fragilidade, foi utilizado o instrumento Probability of Repeated Admission With 4 Years (Probabilidade de admissão hospitalar repetida), proposto por Boult et al. (1993). Os estratos de risco de fragilidade seguem os propostos no estudo de Veras (2003) para a realidade brasileira. A distribuição dos níveis de estratificação de risco, segundo a probabilidade de admissão hospitalar repetida utilizada, foi a seguinte: $<0,300$ (idoso estratificado como sendo de baixo risco), 0,300-0,399 (idoso estratificado como sendo de médio risco), 0,400-0,4990 (idoso estratificado como sendo de risco médio-alto) e $\geq 0,500$ (idoso estratificado como sendo de alto risco).

Para análise da realização e orientações sobre os exames preventivos dos cânceres de mama e de colo do útero, foram analisadas as seguintes variáveis: realização do autoexame de mama, exame clínico de mama, mamografia e exame citopatológico do colo do útero.

O tratamento estatístico dos dados foi realizado com o auxílio do software estatístico IBM SPSS 20. A análise descritiva dos dados será apresentada por meio de tabelas de distribuição de frequência e de contingência. Para a obtenção do grau de fragilidade, foi utilizada uma técnica estatística de análise multivariada chamada regressão logística binária. Para o estudo de associação entre as variáveis qualitativas, foi aplicado o teste Qui-Quadrado ou o teste exato de Fisher (para valores esperados menores que 5). Um valor de $\mathrm{p} \leq 0,05$ foi considerado estatisticamente significativo.

\section{Resultados}

Aidade das idosas foi estratificada em quatro faixas, conforme a seguinte distribuição: $183(53,4 \%)$ dos 60 aos 69 anos, $97(28,3 \%)$ dos 70 aos 79 anos, $55(16 \%)$ dos 80 aos 89 anos e $8(2,3 \%)$ com 90 anos ou mais. Com relação às características sociodemográficas, $261(76,09 \%)$ idosas eram aposentadas, $141(41,1 \%)$ eram casadas e $138(40,23 \%)$ viúvas, $210(61,22 \%)$ relataram renda de 1 salário mínimo, 236 $(68,80 \%)$ não mantinham convênio de saú- 
de e $220(64,13 \%)$ relataram ter apenas 0 ensino fundamental incompleto (Tabela 1).

Tabela 1: Caracterização da amostra de idosas de Cruz Alta - RS, $2012(\mathrm{n}=343)$

\begin{tabular}{|c|c|c|}
\hline Característica & $\begin{array}{r}\text { Frequência } \\
\text { absoluta }(n)\end{array}$ & $\begin{array}{l}\text { Frequência } \\
\text { relativa (\%) }\end{array}$ \\
\hline \multicolumn{3}{|l|}{ Estado civil } \\
\hline Casado & 141 & 41,11 \\
\hline Solteiro & 43 & 12,54 \\
\hline Viúvo & 138 & 40,23 \\
\hline Separado & 21 & 6,12 \\
\hline \multicolumn{3}{|l|}{ Renda (salários mínimos) } \\
\hline Sem renda & 30 & 8,75 \\
\hline 1 & 210 & 61,22 \\
\hline 2 a 5 & 90 & 26,24 \\
\hline 6 a 10 & 3 & 0,87 \\
\hline+ de 10 & - & - \\
\hline Não informado & 10 & 2,92 \\
\hline \multicolumn{3}{|l|}{ Escolaridade } \\
\hline Analfabeto & 39 & 11,37 \\
\hline $\begin{array}{l}\text { Ensino fundamental } \\
\text { incompleto }\end{array}$ & 220 & 64,13 \\
\hline $\begin{array}{l}\text { Ensino fundamental } \\
\text { completo }\end{array}$ & 45 & 13,12 \\
\hline Ensino médio completo & 30 & 8,75 \\
\hline Ensino superior completo & 8 & 2,33 \\
\hline Pós-graduação & 1 & 0,30 \\
\hline Total & 343 & 100,00 \\
\hline
\end{tabular}

Fonte: dados da pesquisa.

Quanto ao grau de fragilidade, segundo o risco de internações repetidas e demais fatores, e por meio da técnica de regressão logística binomial, os resultados demonstraram que a maioria das idosas apresentou risco baixo de adoecer, como demonstrado na Tabela 2.
Tabela 2: Estratificação do grau de fragilidade em idosas de Cruz Alta - RS, 2012

\begin{tabular}{l|r|r}
\hline Grau de Fragilidade & Frequência $(\mathrm{n})$ & Frequência (\%) \\
\hline Risco baixo & 213 & 62,10 \\
Risco médio & 28 & 8,16 \\
Risco médio-alto & 54 & 15,74 \\
Risco alto & 48 & 14,00 \\
Total & 343 & 100,00 \\
\hline \multicolumn{2}{l}{ Fonte: dados da pesquisa. }
\end{tabular}

A associação entre os fatores de risco e o grau de fragilidade está representada na Tabela 3. Pode-se observar que as idosas na faixa etária entre 60 e 69 anos foram as que mais apresentaram baixo risco de adoecer, representando 59,20\% do total do grupo, foi também o grupo que apresentou maior percentual de idosas com alto risco de adoecer, 41,70\% do total. Essa faixa etária também apresentou o maior número de idosas entrevistadas. Quanto à percepção de saúde, $34(70,80 \%)$ idosas, com alto risco de adoecer, relataram apresentar uma saúde média, enquanto 94 (74,00\%) idosas, com baixo risco de adoecer, alegaram que sua saúde era boa, além de $100 \%$ das idosas com baixo risco (213 idosas) relatarem não terem sido internadas nenhuma vez nos últimos doze meses, apesar de 27,69\% (59 idosas) desse grupo, terem procurado o médico por pelo menos uma vez nos últimos doze meses. Ainda, quando perguntadas se apresentavam alguma patologia, apenas 5,20\% (11) afirmaram ter doença cardíaca e $16,90 \%$ (56) informaram Diabetes mellitus. De outro modo, no grupo de alto risco, 35,40\% (17) tinham doença cardíaca e $37,50 \%$ (30) Diabetes mellitus. Tanto no grupo de baixo risco como no de alto risco, as idosas afirmaram ter apoio social de amigo, parente ou vizinho caso necessitassem, $92,50 \%$ (197) e $95,80 \%$ (45), respectivamente. 
Tabela 3: Relação dos fatores de risco com o grau de fragilidade de idosas de Cruz Alta - RS, 2012

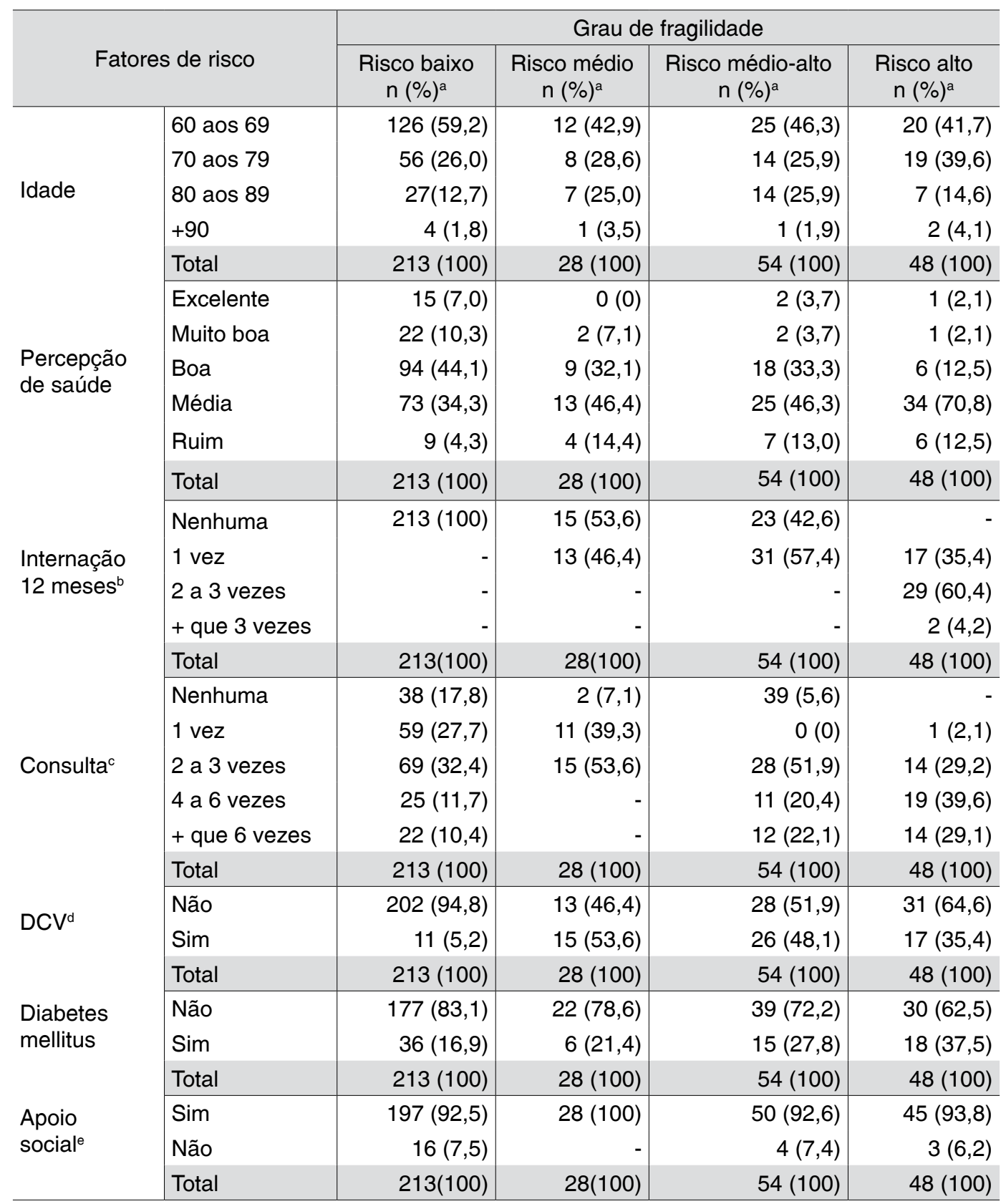

Fonte: dados da pesquisa.

Nota: ${ }^{a}$ Porcentagem referente ao total do grupo de grau de fragilidade. ${ }^{b}$ Hospitalização nos 12 meses anteriores à entrevista. ${ }^{c}$ Número de consultas médicas nos 12 meses anteriores à entrevista. d Doença cardiovascular: angina,

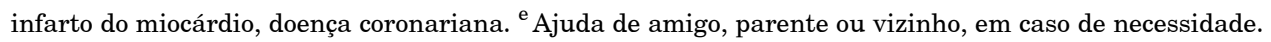


Quanto à orientação da importância da realização dos exames preventivos dos cânceres do colo do útero e da mama, $273(79,59 \%)$ das idosas entrevistadas relataram ter recebido orientação sobre o assunto, enquanto $70(20,41 \%)$ idosas não receberam informação alguma. Dentre as 273 idosas que receberam orientação, as instruções foram dadas, na maioria dos casos, pelo médico, $186(68,13 \%)$, pelo agente de saúde, $47(17,22 \%)$, por profissionais de enfermagem, $19(6,96 \%)$, e por outros profissionais da saúde, 21 (7,69\%).

Entretanto, considerando a realização desses exames, apenas $84(24,49 \%)$ das idosas fizeram o exame Papanicolau há menos de um ano, $161(46,94 \%)$ há dois anos ou mais, e $98(28,57 \%)$ alegaram nunca ter realizado esse exame. Ainda, 164 (47,81\%) idosas afirmaram ter feito o autoexame das mamas há menos de um ano, $49(14,29 \%)$ há dois anos ou mais, e $130(37,90 \%)$ afirmaram que nunca o fizeram. Com relação ao exame clínico das mamas, 122 (35,57\%) idosas relataram sua realização há menos de um ano, $90(26,24 \%)$ há dois anos ou mais, e $131(38,19 \%)$ nunca o realizaram. Já a mamografia, foi feita por $108(31,19 \%)$ idosas há menos de um ano, $101(29,44 \%)$ há dois anos ou mais, e $134(39,07 \%)$ idosas nunca o realizaram (Tabela 4).
Tabela 4: Realização dos exames preventivos dos cânceres do colo do útero e mama por idosas de Cruz Alta - RS

\begin{tabular}{l|r|r}
\hline \multicolumn{1}{c|}{ Exame } & $\begin{array}{r}\text { Frequência } \\
\text { absoluta (n) }\end{array}$ & $\begin{array}{r}\text { Frequência } \\
\text { relativa (\%) }\end{array}$ \\
\hline Papanicolau & & \\
Menos de 1 ano & 84 & 24,49 \\
2 anos ou mais & 161 & 46,94 \\
Nunca fez & 98 & 28,57 \\
Total & 343 & 100,00 \\
\hline Autoexame de mama & & \\
Menos de 1 ano & 164 & 47,81 \\
2 anos ou mais & 49 & 14,29 \\
Nunca fez & 130 & 37,90 \\
\hline Total & 343 & 100,00 \\
\hline Exame clínico de & & \\
mama & 122 & 35,57 \\
Menos de 1 ano & 90 & 26,24 \\
2 anos ou mais & 131 & 38,19 \\
\hline Nunca fez & 343 & 100,00 \\
\hline Total & & \\
\hline Mamografia & 108 & 31,49 \\
Menos de 1 ano & 101 & 29,44 \\
2 anos ou mais & 343 & 39,07 \\
\hline Nunca fez & & 100,00 \\
\hline Total & & \\
\hline
\end{tabular}

Fonte: dados da pesquisa.

Não foi encontrada a associação estatisticamente significativa entre as variáveis grau de fragilidade e exames preventivos realizados pelas idosas (Papanicolau, $\mathrm{p}=0,645$, autoexame de mama, $\mathrm{p}=0,764$, exame clínico de mamas, $p=0,983$, e mamografia, $p=0,992$ ), ou seja, embora as idosas tivessem realizado esses exames preventivos, elas também apresentaram algum quadro de fragilidade (Tabela 5). 
Dentre idosas com alto risco, $68,6 \%$ (10 idosas há menos de um ano e 23 em dois anos ou mais) não deixaram de realizar o Papanicolau, 62,6\% (21 idosas há menos de um ano e nove em dois anos ou mais) realizaram o autoexame das mamas, 60,4\% (dezesseis idosas há menos de um ano e treze em dois anos ou mais) alegaram ter feito o exame clínico e a mamografia (Tabela 5).

Tabela 5: Relação do risco de fragilidade e realização de exames preventivos por idosas de Cruz Alta - RS, 2012

\begin{tabular}{|c|c|c|c|c|c|}
\hline \multirow{3}{*}{ Exame } & \multirow{3}{*}{ Última realização } & \multicolumn{4}{|c|}{ Grau de fragilidade } \\
\hline & & Risco baixo & Risco médio & Risco médio-alto & Risco alto \\
\hline & & n (\%) & n (\%) & n (\%) & n (\%) \\
\hline \multirow{4}{*}{ Papanicolau } & Menos de 1 ano & $55(25,8)$ & $4(14,3)$ & $15(27,8)$ & $10(20,8)$ \\
\hline & 2 anos ou mais & $95(44,6)$ & $15(53,6)$ & $28(51,8)$ & $23(47,8)$ \\
\hline & Nunca fez & $63(29,6)$ & $9(32,1)$ & $11(20,4)$ & $15(31,4)$ \\
\hline & Total & $213(100)$ & $28(100)$ & $54(100)$ & $48(100)$ \\
\hline \multirow{4}{*}{$\begin{array}{l}\text { Autoexame de } \\
\text { mama }^{b}\end{array}$} & Menos de 1 ano & $107(50,2)$ & $10(35,7)$ & $26(48,1)$ & $21(43,8)$ \\
\hline & 2 anos ou mais & $28(13,1)$ & $4(14,3)$ & $8(14,8)$ & $9(18,8)$ \\
\hline & Nunca fez & $78(36,6)$ & $14(50,0)$ & $20(37,0)$ & $18(37,5)$ \\
\hline & Total & $213(100)$ & $28(100)$ & $54(100)$ & $48(100)$ \\
\hline \multirow{4}{*}{$\begin{array}{l}\text { Exame clínico de } \\
\text { mamac }^{c}\end{array}$} & Menos de 1 ano & $77(36,2)$ & $9(32,1)$ & $20(37,0)$ & $16(33,3)$ \\
\hline & 2 anos ou mais & $53(24,8)$ & $8(28,6)$ & $16(29,7)$ & $13(27,1)$ \\
\hline & Nunca fez & $83(39,0)$ & $11(39,3)$ & $18(33,3)$ & $19(39,6)$ \\
\hline & Total & $213(100)$ & $28(100)$ & $54(100)$ & $48(100)$ \\
\hline \multirow{4}{*}{ Mamografia $^{d}$} & Menos de 1 ano & $67(31,5)$ & $7(25,0)$ & $18(33,3)$ & $16(33,3)$ \\
\hline & 2 anos ou mais & $63(29,5)$ & $9(32,1)$ & $16(29,7)$ & $13(27,1)$ \\
\hline & Nunca fez & $83(39,0)$ & $12(42,9)$ & $20(37,0)$ & $19(39,6)$ \\
\hline & Total & 213(100) & $28(100)$ & $54(100)$ & $48(100)$ \\
\hline
\end{tabular}

Fonte: Dados da pesquisa.

Nota: ${ }^{\mathrm{a}} \chi^{2}=4,235 ; p=0,645 \quad{ }^{\mathrm{b}} \chi^{2}=3,346 ; p=0,764 \quad{ }^{\mathrm{c}} \chi^{2}=1,070 ; p=0,983 \quad{ }^{\mathrm{d}} \chi^{2}=0,810 ; p=0,992$.

\section{Discussão}

A Organização Mundial de Saúde (OMS) considera idoso o indivíduo que apresenta 65 anos ou mais. Porém, neste estudo, foi adotado o que é preconizado no Estatuto do Idoso (2003), nas Diretrizes Legais da Política Nacional de Saúde da Pessoa Idosa, que define como idoso o indivíduo de 60 anos ou mais (BRASIL, 2006).
Segundo Oliveira e Menezes (2011), a idade avançada é um fator de risco para a fragilidade devido às perdas fisiológicas comuns do processo de envelhecimento do organismo e o acúmulo de fatores de risco. No presente estudo, as idosas avaliadas foram classificadas, em sua grande maioria, dentro do grau de risco baixo de adoecer. Mesmo entre aquelas da faixa etária de 90 anos ou 
mais, $50 \%$ das idosas ficaram no grupo de baixo risco.

Em relação aos fatores de risco com o grau de fragilidade, pode-se observar que a faixa etária entre 60 e 69 anos foi a que mais apresentou idosas com risco baixo e também de alto risco $(59,2 \%$ e $41,7 \%$, respectivamente). Porém, cabe ressaltar que essa faixa etária apresentou o maior número de idosas entrevistadas, o que pode ter contribuído para tais achados. Quanto à percepção de saúde, o percentual mais elevado no grupo de risco baixo foi o de saúde boa, nos demais grupos (médio, médio-alto e alto) foi o de saúde média. Nota-se, portanto, que além do fato da fragilidade desses grupos, as idosas percebem-se frágeis, juntando efeitos físicos aos efeitos psicossociais (NERI, 2001), pois essas idosas também alegaram apresentar alguma doença cardíaca e Diabetes mellitus.

As últimas estimativas do Instituto Nacional do Câncer (INCA, 2013) apontam para 57 mil e 15 mil novos casos de câncer de mama e câncer de colo do útero, respectivamente. Mesmo diante de dados alarmantes, uma boa porcentagem das idosas entrevistadas relatou nunca ter feito algum tipo de exame preventivo. Foi identificado que uma proporção significativa nunca realizou o Papanicolau, o autoexame de mama, o exame clínico das mamas ou mamografia.

Segundo Fonsêca, Godoi e Silva (2010), mulheres com benefícios governamentais apresentam os indicadores mais altos de prática adequada em relação aos exames. Quanto à periodicidade da realização, descrevem que as mulheres que realizam frequentemente os exames, como preconiza o Ministério da Saúde, associam a ida ao médico a um ato de cuidado com sua saúde. Entretanto, as mulheres que não mantêm uma frequência associam a realização com alguma patologia já existente. Enquanto uma parcela muito pequena da população procura o serviço de saúde para fazer exclusivamente a prevenção.

A baixa procura por tais exames é uma realidade entre a terceira idade. No município de Cruz Alta, no mês de janeiro de 2013, foram realizados 211 exames de Papanicolau, dos quais, apenas $21 \mathrm{em}$ mulheres acima de 60 anos (INCA, 2012a). O estudo de Iwamoto et al. (2011) demonstrou que a idade média das mulheres que fizeram exames Papanicolau nas Estratégias de Saúde da Família do município de Novo Cruzeiro, Minas Gerais, foi de 30 anos, com predomínio da faixa etária entre 25 e 44 anos. O notável foi que, entre as mulheres com 60 anos ou mais, a prevalência foi muito baixa, apenas $8,32 \%$ realizaram o exame.

O mesmo acontece em relação aos exames preventivos do câncer de mama. Dados do Sistema de Informação do Câncer de Mama (Sismama), levantados no período de janeiro a abril de 2013, mostraram que foram realizadas 721.786 mamografias no Brasil, dessas, somente $179.318 \mathrm{em}$ mulheres acima de 60 anos. Comparando esses dados com os do mesmo período do ano de 2012 , nota-se uma grande discrepância, pois foram realizadas 1.147.797 mamografias, das quais 273.623 foram exames em mulheres com mais de 60 anos (INCA, 2012b). 
As recomendações do Ministério da Saúde, para ambos os cânceres, mama e colo do útero, são bem claras e estão difundidas entre a população pesquisada, pois $79,89 \%$ das idosas entrevistadas relataram ter recebido orientação sobre o assunto. Mesmo assim, foi observado um percentual relativamente elevado de não realização dos exames preventivos. Oliveira et al. (2004) afirmam que um dos principais fatores que interferem na realização de exames para diagnóstico precoce é, sem dúvida, a descrença das mulheres com relação ao processo da prevenção.

No Brasil, preconiza-se para o rastreamento do câncer de colo do útero, a realização do exame Papanicolau a partir dos 25 anos de idade para as mulheres que já tiveram atividade sexual, até os 64 anos, com intervalo entre os exames inicialmente anual, e, após dois resultados negativos consecutivos, deverá ser feito com intervalo de três anos (INCA, 2011).

Já para a detecção precoce do câncer de mama, o Ministério da Saúde preconiza o rastreamento por meio do exame clínico da mama para todas as mulheres a partir de 40 anos de idade, realizado anualmente, e o rastreamento por mamografia para as mulheres com idade entre 50 e 69 anos, com o máximo de dois anos entre os exames (INCA, 2004).

Tendo em vista que as orientações do Ministério da Saúde chegam até a comunidade principalmente por meio da educação em saúde, Fonsêca, Godoi e Silva (2010) citam em seu estudo diversos autores que mencionaram os demais profissionais da saúde como contribuintes para o aprimoramento na educação em saúde da população, ao mesmo tempo em que diminuem a sobrecarga médica. Além disso, salientam que esses profissionais devem garantir a atenção primária, trabalhando para minimizar os fatores de risco, ensinar atitudes preventivas e de promoção da saúde, conscientizando e incentivando a população a essas práticas.

Outro fato merecedor de atenção é o citado por Santos e Chubaci (2011), no que se refere às atitudes relacionadas ao corpo, como o autoexame da mama, as quais, segundo os autores, permeiam fatores culturais, tabus, valores e crenças da mulher. Um estudo realizado em Botucatu apresentou um percentual mais elevado da realização do autoexame das mamas por mulheres mais jovens, em comparação com as mais velhas, o que demonstra a necessidade de atividades de educação em saúde sobre essa temática para o público da terceira idade (MOLINA; DALBEN; DE LUCA, 2003).

A análise estatística dos dados demonstrou que não há associação estatisticamente significativa entre 0 grau de fragilidade e a realização dos exames preventivos, ou seja, uma idosa fragilizada não necessariamente deixará de realizar os exames preventivos dos cânceres de colo do útero e de mama. Isso porque, considerando apenas as idosas com risco alto de adoecer, a maioria afirmou realizar o exame Papanicolau, o autoexame de mama, o exame clínico de mama e a mamografia.

De certa forma, os achados relacionam-se com o estudo de Santos e Chubaci (2011), que indica que o co- 
nhecimento é fator fundamental para realizar a mamografia $(p=0,047)$, visto que as idosas entrevistadas receberam orientação e realizaram a mamografia e os demais exames. Mesmo assim, as taxas de realização dos exames preventivos dos cânceres de colo do útero e de mama apresentados pelo Sismama, no município de Cruz Alta, estão abaixo do esperado.

\section{Conclusão}

Não foi encontrada associação significativa entre as variáveis grau de fragilidade e a de exames preventivos dos cânceres de mama e de colo do útero realizados pelas idosas. Mesmo assim, é importante a continuidade e a ampliação das estratégias de educação em saúde sobre a detecção precoce dessas doenças, sobretudo na população idosa, de forma a garantir a adesão dessas mulheres a esse cuidado preventivo. Certamente essas neoplasias são temas em evidência no cenário nacional. No entanto, é visível que os cuidados com a população idosa ainda constituem desafio para o sistema de saúde brasileiro. A fragilidade decorrente da senilidade e as mudanças celulares provocadas pelo envelhecimento aumentam a suscetibilidade de desenvolvimento de neoplasias nos idosos.

Um resultado satisfatório encontrado no estudo foi de que a maioria das idosas entrevistadas tem informação sobre os exames preventivos dos cânceres de colo do útero e de mama. Porém, foi observado que ainda existem idosas que não se submetem periodicamente aos exames preventivos ou nunca os realizaram.
Assim, é importante a continuidade e a ampliação das estratégias de educação em saúde sobre a detecção precoce dessas doenças, sobretudo na população idosa, de forma a garantir a adesão dessas mulheres a esse cuidado preventivo.

Ressalta-se também, que é importante e necessária a realização de novos estudos relacionando a realização de exames preventivos com o grau de fragilidade em mulheres idosas. Pois, se o baixo índice de realização de exames na população idosa não está relacionado com o grau de fragilidade que ela apresenta, seria necessário avaliar as demais variáveis, a fim de que as prevalências de realização dos exames preventivos dos cânceres de colo do útero e de mama, no futuro, sejam mais elevadas. Portanto, o presente estudo seria mais uma contribuição para as pesquisas na área da gerontologia e da oncologia, além de um estímulo para o desenvolvimento de futuros trabalhos na área.

\section{Relationship between the elderly frailty and achievement of preventive exams}

\section{Abstract}

The elderly population is the part that grows the most in the Brazil, as around the world and, the front of senility, the prevention against the breast and cervical cancer can't be discarded, further if addressing of frail elderly, due to their clinical condition unfavorable. To verify significant association between the fragility degree and preventive examinations of cancer of breast and cervical cancer by the elderly, we aim at this work. The data were collected by structured interview with elderly aged 60 
or over that live in the city of Cruz Alta. The instrument aims at considering the examined variables: forms containing sociodemographic data, early detection and predictability of grievances and exams. The data analysis was realized using the software IBM SPSS 20. The studied sample was composed by 343 women with $62.1 \%$ of elderly presented low risk of getting sick, $8.16 \%$ medium risk, $15.74 \%$ medium-high risk and $14 \%$ high risk. Within the recommendations, $79.59 \%$ of elderly received some directions about the importance of getting a preventive examinations, while $28.57 \%$ had never taken a Papanicolau's test, $37.90 \%$ have never done a breast self-examination, $38.19 \%$ had never performed a clinical breast exam and $39.07 \%$, a mammography. It wasn't observed any significant association between the frailty and preventive examinations performed by elderly. However, it is important the continuity and extension of education strategies on health related to early detection of these diseases, mainly on the elderly population to guarantee that these women will have access to this preventive care.

Keywords: Elderly. Fragility. Prevention. Mammography. Papanicolau.

\section{Agradecimentos}

Os autores agradecem às idosas participantes do estudo, que gentilmente concordaram em participar da pesquisa, a fim de colaborar com a ciência. Às agentes comunitárias de saúde do município de Cruz Alta também o nosso agradecimento, por auxiliarem nas visitas, e à Secretaria Municipal de Saúde de Cruz Alta, que deu suporte para a realização do estudo. Da mesma forma, agradecemos ao financiamento obtido no edital PPSUS/Fapergs que possibilitou financeiramente a realização da pesquisa.

\section{Referências}

BRASIL. Ministério da Saúde. PORTARIA GM/2.528 DE 19 DE OUTUBRO DE 2006. Brasília, Ministério da Saúde. 2006.

Atenção à saúde da pessoa idosa e envelhecimento. 2010. Disponível em: <http://bvsms.saude.gov.br/bvs/publicacoes/ atencao_saude_pessoa_idosa_envelhecimento_v12.pdf>. Acesso em: 10 maio 2013.

BOULT, C. et al. Screening elders for risk of hospital admission. Journal of the American Geriatrics Society, v. 41, n. 8, p. 811-7, Aug. 1993.

DA SILVA, M. M.; DA SILVA, V. H. Envelhecimento: importante fator de risco para o câncer. Arquivos Médicos do ABC, São Paulo, v. 30 , n. 1, p. 11-18, jan./jul. 2005.

FONSÊCA, W.; GODOI, S.; SILVA, J. V. B. Papanicolaou na terceira idade: conhecimento e atitude das idosas cadastradas em uma Estratégia de Saúde da Família da cidade de Itaporã - MS. Revista Brasileira de Ciências do Envelhecimento Humano, Passo Fundo, v. 7, n. 3, p. 357-369, 2010.

IBGE - INSTITUTO BRASILEIRO DE GEOGRAFIA E ESTATÍSTICA. Diretoria de Pesquisas, Coordenação de Trabalho e Rendimento, Pesquisa Nacional por Amostra de Domicílios. Resultados do Censo de 2010. Disponível em: <http://www.censo2010.ibge.gov. br/es/resultados>. Acesso em: 27 abr. 2013.

INCA - INSTITUTO NACIONAL DE CÂNCER. Controle do câncer de mama. Documento de consenso. Rio de Janeiro: INCA, 2004.

. Diretrizes brasileiras para o rastreamento do câncer do colo do útero. Rio de Janeiro: INCA, 2011.

Estimativa 2014 - Incidência de câncer no Brasil. Rio de Janeiro: INCA, 2013.

Sistema de Informação do Câncer do Colo do Útero-Siscolo. Rio de Janeiro: INCA, 2012a. 
. Sistema de Informação do Câncer de Mama - Sismama. Rio de Janeiro: INCA, 2012b.

IWAMOTO, H. H. et al. Mulheres que realizam Papanicolaou: contribuições para a estratégia saúde da família. Cogitare Enfermagem, Paraná, v. 16, n. 3, p. 424-429, 2011.

MOLINA, L; DALBEN, I; DE LUCA, L. Análise das oportunidades de diagnóstico precoce para as neoplasias malignas de mama. $R e-$ vista da Associação Médica Brasileira, São Paulo, v. 49, n. 2, p. 185-190, 2003.

NERI, A. L. Envelhecimento e qualidade de vida na mulher. In: CONGRESSO PAULISTA DE GERIATRIA E GERONTOLOGIA, 2, 2001, São Paulo. Anais... Disponível em: <http://www.portaldoenvelhecimento.org.br/ artigos/maio2007/2congresso.pdf $>$. Acesso em 25 abr. 2013.

OLIVEIRA, L. P.; MENEZES, R. M. P. Representações de fragilidade para idosos no contexto da estratégia saúde da família. Texto contexto - enfermagem, Florianópolis, v. 20, n. 2, p. 301-309, abr./jun. 2011.

OLIVEIRA, M. M. et al. Câncer cérvicouterino: um olhar crítico sobre a prevenção. $R e$ vista Gaúcha de Enfermagem, Porto Alegre, v. 25 , n. 2, p. 176-183, 2004.

SANTOS, G. D; CHUBACI, R. Y. S. O conhecimento sobre o câncer de mama e a mamografia das mulheres idosas frequentadoras de centros de convivência em São Paulo (SP, Brasil). Ciência \& Saúde Coletiva, São Paulo, v. 16, n. 5, p. 2533-2540, maio 2011.

SILVESTRE, J. A.; COSTA NETO, M. M. Abordagem do idoso em programas de saúde da família. Cadernos de Saúde Pública, Rio de Janeiro, v. 19, n. 3, p. 839-847, 2003.

TRIBESS, S.; OLIVEIRA, J. R. Síndrome da fragilidade biológica em idosos: revisão sistemática. Revista de Salud Pública, São Paulo, v. 13, n. 5, p. 853-864, out. 2011.

VERAS, R. P. Em b usca de uma assistência adequada à saúde do idoso: revisão da literatura e aplicação de um instrumento de detecção precoce e de previsibilidade de agravos. Caderno de Saúde Pública, Rio de Janeiro, v. 19, n. 3, p. 705-715, jan./jun. 2003.

VERAS, R. P. Experiências e tendências internacionais de modelos de cuidado para com o idoso. Ciência \& Saúde Coletiva, Rio de Janeiro, v. 17, n. 1, p. 231-238, jan. 2012. 